\title{
Mapping school-wide nutrition \& physical activity practices: Results from student participation with a new research tool
}

\author{
S. Browne ${ }^{1}$, C. Barron ${ }^{1}$, V. Lambert ${ }^{1}$, D. Susta ${ }^{2}$, A. Staines ${ }^{1}$ and M.R. Sweeney ${ }^{1}$ \\ ${ }^{1}$ NURISH School Nursing \& Human Sciences and ${ }^{2}$ School Health \& Human Performance, Dublin City University, \\ Dublin 9, Republic of Ireland ( ROI)
}

Secondary school students consume a significant proportion of their daily calorie intakes and may expend over half of their daily energy during school hours ${ }^{1}$. Student input into food and physical activity (PA) facilities at school is at best minimal. The aim of this study was to develop a tool that students could use to explore the food and PA cultures of secondary schools in the Republic of Ireland (ROI). It is part of a larger research project examining factors that influence the diet and PA behaviours of adolescents.

Data from youth panel discussions, literature reviews, EU and national policy documents and guidelines were combined to develop a set of questions that would adequately examine the food and PA environments of secondary schools. A pilot study was undertaken in two schools with a two-pronged School Environment Observation Tool (SEOT) after which required adjustments were made. Six secondary schools from different socio-economic and geographical areas, and representing both genders, participated in the final study. A researcher instructed a total of 28 transition year students (15-17 years old) on how to use the SEOT to take notes and photographs over one typical school day. Student researchers were asked to fill in an evaluation questionnaire about their participation.

Notes and photographs collected by students in this study show that food and physical activity cultures and practices vary greatly by school. The food consumption of students at school depends upon many factors including food available in the school shop or canteen, the presence of vending machines, permission to leave the school grounds at lunch time, the types of food outlets nearby, access to food storage and heating facilities, breakfast club facilities, food brought from home or other places, and school food policies. Factors affecting PA participation at school include the range of sports facilities on schools grounds, the allocation of weekly PE classes, the range of extra-curricular sports on offer, and availability of sports equipment for students during break times. Transport options to and from school varied, with urban school students more likely to walk or cycle than rural school students. The duration of lunch break, which ranged from 40 to 60 minutes, seems to influence both food consumption and PA participation.

Results from the student evaluation questionnaires showed that they felt confident about undertaking the research. Many reported that they enjoyed the methods and discovering new things about typical student behaviour and school facilities.

"I have learned new ways to research about activities" (Male, School 4)

"I realise I should eat healthy and exercise more after my experience" (Female, School 6)

This study is of benefit for two reasons. The methodology engaged students in research and, through data collection, raised their awareness of the topic. It can be viewed as the first stage in a participatory research approach that actively involves students in school health promotion ${ }^{2}$. Secondly, future research will examine the relationship between the SEOT results and student food consumption, PA patterns, and anthropometry and fitness data. Findings will be used to inform the development of nutrition and PA scales for ROI secondary schools that will help guide healthy eating and physical activity policies and practice.

1. Briefel RR et al. (2009) JADA 109(2), S91-S107.

2. Griebler MU, Rojatz MD, Simovska V, Forster R (2012) Evidence for the effects of student participation in designing, planning, implementing and evaluating school health promotion - a systematic literature review. Working Paper No. 12 of the LBIHPR. 